\title{
Fluconazole Susceptibility and Genotypic Heterogeneity of Oral Candida albicans Colonies from the Patients with Cancer Receiving Chemotherapy in China
}

\author{
Jing Sun ${ }^{1}$, Cheng $\mathbf{Q i}^{1,2}$, Micheal D. Lafleur ${ }^{3}$, Qing-guo $Q i^{1 *}$ \\ ${ }^{1}$ Department of Oral Medicine, Key Lab of Oral biomedicine Shandong Province, School of Stomatology Shandong \\ University, Jinan, China \\ ${ }^{2}$ The Secondary Hospital affiliated to Shandong University, Jinan, China \\ ${ }^{3}$ Antimicrobial Discovery Center and Department of Biology, Northeastern University, Boston, USA
}

\begin{abstract}
Jing Sun, Cheng Qi, Micheal D. Lafleur, Qing-guo Qi. Fluconazole Susceptibility and Genotypic Heterogeneity of Oral Candida albicans Colonies from the Patients with Cancer Receiving Chemotherapy in China. International Journal of Oral Science, 1(3): 156-162, 2009
\end{abstract}

\begin{abstract}
Aim To identify heterogeneity of Candida albicans ( $C$. albicans) isolated from the population with cancer in China by using identification medium, subculture molecular typing, and antifungal susceptibility test.
\end{abstract}

Methodology Oral cheek mucosal specimens from 52 cancer patients receiving chemotherapy were cultured on CHROMagar Candida ${ }^{\mathrm{TM}}$ plates for Candida identification. All the C.albicans colonies on the plates were subcultured and reconfirmed by API20C, then submitted to the antifungal drug susceptibility test with fluconazole and molecular typing using randomly amplified polymorphic DNA-PCR (RAPD) with primers RSD6 and RSD12.

\begin{abstract}
Results 54\% (28/52) patients were oral yeast carriage in which C. albicans predominated. More than 7 C. albicans colonies were isolated from each of 12 patients (Group A), while less than 5 colonies were isolated from each of 16 patients (Group B). RSD6 and RSD12 were successful in eliciting 17 (A1-A17) and 2 (B1-B2) genotypes, respectively from among the 205 isolates. The two primers were combined to generate 21 genotypes. The $C$. albicans isolates obtained from the same patient and episode showed a diversity for fluconazole revealed by $\mathrm{MIC}_{50}$ and $\mathrm{MIC}_{90}$.

Conclusion The heterogeneity of the $C$. albicans colonies isolated from the same patients can be detected. C. albicans with varied fluconazole susceptibility and genotypic characteristics may coexist in the same oral Candida population.
\end{abstract}

Keywords Candida albicans, molecular typing, chemotherapy, antifungal susceptibility
Document code: A

CLC number: R780.2

Received May 5, 2009; Revision accepted Aug. 10, 2009

\section{Introduction}

Candida species especially Candida albicans (C. albicans) are the composition of oral commensal flora, which can be isolated in the healthy oral cavities with different frequency (Cannon et al., 1995; Kleinegger et al., 1996; Qi et al., 2005). At the same time, $C$. albicans is one of the most pervasive opportunistic pathogens, which can cause endogenous infection from superficial to seriously deep-seated mycoses under the altered host conditions, such as the malignant cancer patients (Brawner and Cutler, 1989; McCullough et al., 1996). Anticancer treatment including chemotherapy or/and radiotherapy is still associated with several side effects. Among them, oral candidiasis is one major source of illness, although strict oral hygienic care has been undertaken by using anti- 
fungal reagents to prevent them (Wingard, 1994). Oral candidiasis is often accompanied by pain, loss of taste, reduction in intake, and weight loss, thereby resulting in treatment delays and necessitating dose reduction (Mead, 2002). Furthermore some studies have shown its potential importance in the development of systemic candidiasis which would be vital for these patients (Rodu et al., 1984; Kontoyiannis and Lewis, 2002).

The main limitation of previous studies about oral Candida is the fact that only single isolates are elected from each patient for each episode. In fact there is heterogeneity among the oral Candida population (all the Candida colonies isolated from the same host) in phenotypic and genotypic aspects (Wimpenny et al., 2000; Stewart and Franklin, 2008). For example, one research showed that up to 32 fold differences in fluconazole minimal inhibitory concentrations (MICs) were detected within isolates recovered at the same AIDS patient and episode (Lopez-Ribot et al., 1999).

Since there is great heterogeneity among the individuals of the oral Candida population, it is reasonable to believe that this randomly selected single isolate cannot represent the whole view of the oral Candida population. Until now, the heterogeneity in the oral C. albicans population across China is still unknown, and many studies of genotype variation and antifungal resistance among isolates from AIDS patients have shown mixed results (Takasuka et al., 1998; Metzgar et al., 1998; Lopez-Ribot et al., 1999).

This research may help us to understand the antifungal drug susceptibility and genotypic characteristics of oral Candida isolated from cancer patients in China, and would assist in getting the accurate susceptibility test in clinical practice.

\section{Material and methods}

\section{Patients selection}

Fifty-two cancer patients from two affiliated teaching hospitals and a cancer hospital of Shandong University Medical School were selected for this study and sampled for oral Candida carriage, including 6 patients with hematological malignancies, and 46 with solid tumors. The patients' ages ranged from 21 to 76 years old, averaging 49.8 years. There were 31 male patients and 21 female patients. The inclusion criteria for the study were previous diagnoses of advanced cancer for more than 1 year and treatment by chemotherapy. The exclusion criteria for this study were significant physical defects, denture wearers, uncontrolled diabetes mellitus, systemic antifungal medication or steroids within the recent 2 weeks. The patients with head and neck cancer receiving radiation therapy were excluded. This study was approved by the Ethics Committee of Shandong University (No. 2008-03) and Northeastern University MA US IRB (No. 09-01-02).

\section{Sampling preparation}

All patients received regular oral hygiene care including $0.2 \%$ chlorhexidine daily. Patients were examined by two physicians, according to the guidelines of oral candidiasis diagnostic standards, for evidence of oral candidiasis (Kleinegger et al., 1996). They were sampled with specimens obtained 8 hours following regular oral hygiene care. The specimens were collected by passing a sterile swab across the inner cheek mucosal surface five times. The procedure was repeated three times in total within a half-hour interval. Swabs were transferred to a sterile test tube containing $500 \mu \mathrm{L}$ sterile phosphate buffered saline (PBS). The tubes containing the swabs were vortexed vigorously for 30 seconds to suspend the samples. After 30 seconds, the swabs were removed, the samples were sedimented by centrifugation and concentrated by resuspending in $50 \mu \mathrm{L}$ PBS. This suspension was spread plated on CHROMagar Candida ${ }^{\mathrm{TM}}$ differential medium, and incubated for 24-48 hours (Worthington and Clarkson, 2002). Colonies were identified as Candida by CHROMagar based on color change and colony morphology of this propriety selective and differential medium (Tamura et al., 2001). Candida strains were confirmed by API20C (BioMerieux France) based on carbon source utilization and other differential biochemical assays according to the manufacturer's directions (Xu et al., 2002). C. albicans species were additionally confirmed by observing germ tube formation induced by serum via microscopy (Mackenzie, 1962). If multiple Candida colonies were present, 
all of the isolates were subculture and chosen for further analysis.

\section{Antifungal susceptibility test}

The $10 \mathrm{mg} \cdot \mathrm{mL}^{-1}$ stock solution of amphotericin B (AMB) (Fisher Scientific, USA) and chlorhexidine (CHX) (Sigma-Aldrich, USA) were prepared in dimethyl sulfoxide. The MIC of clinical isolates was determined by broth microdilution based on the CLSI M27-A2 guidelines (McArthur, 2006). Briefly, cells from an overnight culture grown in yeast peptone dextrose medium were harvested by centrifugation, then being washed twice in sterile PBS and resuspended to $0.5 \times 10^{3}-$ $2.5 \times 10^{3}$ cell $\cdot \mathrm{mL}^{-1}$ in RPMI 1640 (GBICO, USA) medium buffered to $\mathrm{pH} 7.0$ with $0.165 \mathrm{~mol} \cdot \mathrm{L}^{-1}$ MOPS (Sigma-Aldrich, USA). Cells were dispensed into a 96-well microtiter plate at $100 \mu \mathrm{L}$ per well, and antifungals were added by a series of 2 -fold dilutions, $100 \mu \mathrm{L}$ per well in RPMI medium. Plates were incubated at $37^{\circ} \mathrm{C}$ for 48 hours. MICs for fluconazole were determined visually and measured by microplate spectrophotometer (Molecular Device, USA) at $600 \mathrm{~nm}$, prior to the identification of the lowest concentration that would result in $50 \%$ $\left(\mathrm{MIC}_{50}\right)$ and $90 \%\left(\mathrm{MIC}_{90}\right)$ reduction.

\section{Genotypic determinations}

Extraction of $C$. albicans DNA, 1-2 monocolonies from the YPD agar plates was suspended in $200 \mu \mathrm{L}$ of TE buffer in a microcentrifuge tube. DNA extraction was carried out using Promega Wizard DNA Extacyion Kit (Promega, USA). Nucleic acid pellet was obtained, washed with ice-cold $70 \%$ ethanol, dried, and resuspended in sterile TE buffer at a concentration of $5 \mu \mathrm{g} \cdot \mathrm{mL}^{-1}$.

Genotypic was determined using randomly amplified polymorphic DNA-PCR (RAPD) analysis. Two primers RSD6 (5'-GCG ATC CCC A-3') and RSD12 (5'-GCA TAT CAA TAA GCG CAG GAA AAG-3') for RAPD were those described by Fell and Akopyanz (Akopyanz et al., 1992; Fell, 1993). Amplification reactions were performed in $50 \mu \mathrm{L}$ of distilled water containing $1 \mu \mathrm{mol} \cdot \mathrm{L}^{-1}$ primer, 200 ng of genomic DNA, and PCR kit including Taq $\left(5 \mathrm{U} \cdot \mu \mathrm{L}^{-1}\right) 0.125 \mu \mathrm{L}, 10 \times$ Ex Taq buffer $5 \mu \mathrm{L}$, $\mathrm{MgCl}_{2}\left(25 \mathrm{mmol} \cdot \mathrm{L}^{-1}\right) 2 \mu \mathrm{L}, 200 \mu \mathrm{mol} \cdot \mathrm{L}^{-1}$ of dNTP mixture, $1.5 \mathrm{U}$ of Taq polymerase (PCR kit of TAKARA, China). The PCR conditions were listed follows: the first five cycles incubated 30 seconds of denaturation at $94^{\circ} \mathrm{C}, 2$ minutes of annealing at $27^{\circ} \mathrm{C}$ (for RSD6) and $52^{\circ} \mathrm{C}$ (for RSD12), and 2 minutes of primer extension at $72^{\circ} \mathrm{C}$, then a final extension at $72{ }^{\circ} \mathrm{C}$ for 15 minutes in a thermoreactor (Gradient Thermal Cycle, Promega Corp, USA).

The amplification products were characterized by electrophoresis on $1.2 \%$ agarose gels in $1 \times$ TBE buffer at $50 \mathrm{~V}$ for 120 minutes, stained in a solution of $0.5 \mu \mathrm{g} \cdot \mathrm{mL}^{-1}$ of ethidium bromide, and then visualized by UV transillumination (Bio Rad, USA) (Waltimo et al., 2001).

\section{Results}

\section{Oral Candida carriage and antifungal suscep- tibility of the Candida isolates from cancer patients}

Oral Candida strains were isolated from 28 of 52 patients and the frequency of oral Candida carriage was $53.8 \%$. Among them, Candida glabrata (C. glabrata) alone was detected in 3 patients.

The fungal burden of the patients was determined using the numbers of yeast colonies on the identification medium of each sample (Rüchel et al., 2000). All the patients were divided into two groups according to the fungal burden. The average number of yeast cfu of Group A (fungal burden more than 7) was $13.4 \pm 5.2(n=12)$, significantly higher than that of Group B (fungal burden less than 5), i.e., $2.8 \pm 1.4(n=16)(P<0.001)$ (table 1).

\section{Heterogeneity of antifungal susceptibility and genotype among the isolates of oral $C$. albicans population}

The two primers, RSD6 and RSD12, were successful in eliciting 17 (A1-A17) and 2 (B1-B2) genotypes, respectively from among the 205 isolates. RSD6 (A1-A17) and RSD12 (B1-B2) were combined to generate 21 genotypes. Multiple $C$. albicans strains isolated from most of the patients $(8 / 12)$ of Group A belong to the same genotype. While for 3 patients, 2 genotypes coexist at the 
same $C$. albicans population. However the oral $C$. albicans population of patient A8 showed highly genotypic heterogeneity (Figure 1). Much fewer stains were isolated from Group B patients, and genotypic heterogeneity could be detected in the C. albicans population of 4 patients (B1, B6, B9 and B12).

The variation in fluconazole susceptibility and genotype of the $C$. albicans isolates from the patients of Group A were analyzed. Firstly, for the isolates from patient A8, great heterogeneity could be observed in both genotype and fluconazole susceptibility (Table 2). Secondly, all the isolates from patients A12 and A3 were genotype A6B1 and A9B2 respectively, having the same level of $\mathrm{MIC}_{90}$ but different $\mathrm{MIC}_{50}$ for fluconazole (Table 3). Thirdly, two genotypes coexist in the same patient C. albicans population, but all the isolates show the same susceptibility to fluconazole, whatever $\mathrm{MIC}_{50}$ or $\mathrm{MIC}_{90}$ (patients A1, A4 and A7). Lastly, multiple isolates from the same patient were of the same genotype, meanwhile showing the same level of fluconazole susceptibility (patients A5, A6, A10 and A11).

Table 1 Overview of fungal burden, clinical evidence of oral candidiasis and genotypic heterogeneity among the oral $C$. albicans isolates

\begin{tabular}{|c|c|c|c|}
\hline $\begin{array}{c}\text { Group } \\
\text { (Patient) }\end{array}$ & Fungal burden & $\begin{array}{c}\text { Clinical diagnosis of } \\
\text { oral candidiasis }\end{array}$ & $\begin{array}{l}\text { C.albicans genotype } \\
\text { RAPD analysis }\end{array}$ \\
\hline A1 & 6 & Yes & A6B2, B7B2 \\
\hline A2 & 16 (C. glabrata) & Yes & \\
\hline A3 & 7 & Yes & A9B1 \\
\hline A4 & 13 & Yes & A9B2, A13B2 \\
\hline A5 & 17 & Yes & A13B1 \\
\hline A6 & 21 & Yes & A10B2 \\
\hline A7 & 18 & yes & A13B9, A14B9 \\
\hline A8 & 10 & Yes & $\mathrm{A} 4 \mathrm{~B} 2, \mathrm{~A} 9 \mathrm{~B} 2, \mathrm{~A} 11 \mathrm{~B} 2, \mathrm{~A} 12 \mathrm{~B} 2, \mathrm{~A} 13 \mathrm{~B} 2$ \\
\hline A9 & 19 & & A15B2 \\
\hline A10 & 7 & & A1B1 \\
\hline A11 & 17 & & A10B2 \\
\hline $\mathrm{A} 12$ & 10 & & A6B1 \\
\hline B1 & 3 & & A1B2, A2B2 \\
\hline B2 & 2 & & A3B2 \\
\hline B3 & 3 (C. glabrata) & Yes & \\
\hline B4 & 4 (C. glabrata) & Yes & \\
\hline B5 & 2 & & A1B2 \\
\hline B6 & 3 & & $\mathrm{~A} 1 \mathrm{~B} 2, \mathrm{~A} 1 \mathrm{~B} 4$ \\
\hline B7 & 1 & & A5B2 \\
\hline B8 & 3 & & A6B2 \\
\hline B9 & 5 & & A3B2, A8B2, A17B2 \\
\hline B10 & 1 & & A9B2 \\
\hline B11 & 2 & & A9B2 \\
\hline B12 & 5 & & A8B2, A9B2 \\
\hline B13 & 4 & & A6B2 \\
\hline B14 & 1 & & A6B2 \\
\hline B15 & 4 & & A4B2 \\
\hline B16 & 1 & & A16B2 \\
\hline
\end{tabular}

The average number of yeast cfu of Group A was $13.4 \pm 5.2(n=12)$, significantly higher than that of Group B $2.8 \pm 1.4$ $(n=16)$. Most of the Group A patients and all of the oral C. glabrata had the clinical evidence of oral candidiasis, indicating that high fungal burden and C. glabrata carriage may be interrelated with oral Candida infectious disease. 


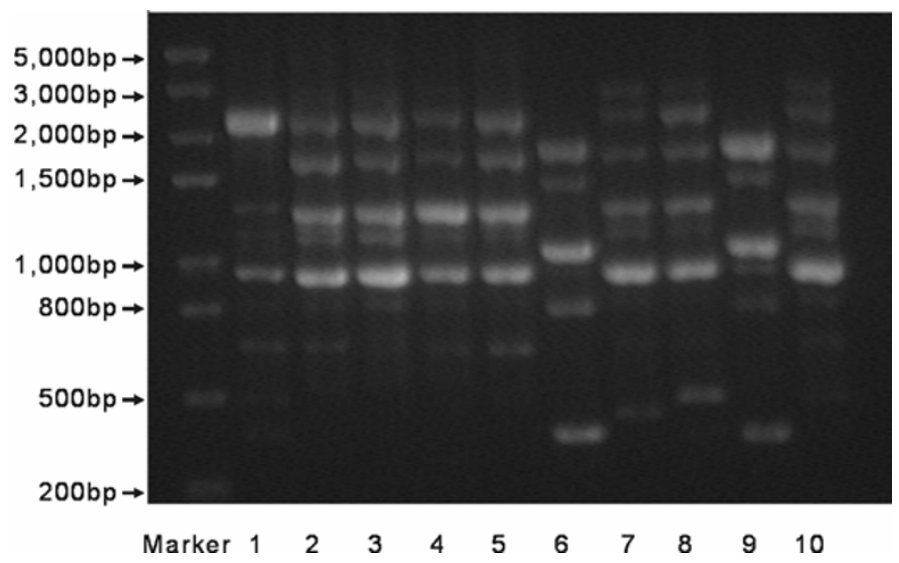

Figure 1 RAPD fingerprints of C. albicans isolated from patient A8 obtained using primer RSD6

Five genotypes were elicited among the 10 strains isolated from patient A8. Please note that No.2, 3, 4, 5, 7 and 8 belong to the same genotype.

Table 2 Heterogeneity in both genotype and fluconazole susceptibility for C. albicans isolated from patient A8

\begin{tabular}{llcc}
\hline Strains & $\mathrm{MIC}_{50} / \mathrm{\mu g} \cdot \mathrm{mL}^{-1}$ & $\mathrm{MIC}_{90} / \mathrm{\mu g} \cdot \mathrm{mL}^{-1}$ & Genotype \\
\hline 1 & 0.5 & 2 & A13B2 \\
2 & 0.125 & 0.5 & A9B2 \\
3 & 0.5 & $>128$ & A9B2 \\
4 & 0.125 & $>128$ & A9B2 \\
5 & 0.125 & $>128$ & A9B2 \\
6 & 0.5 & $>128$ & A11B2 \\
7 & 0.125 & 1 & A9B2 \\
8 & 4 & 16 & A9B2 \\
9 & 0.125 & 4 & A4B2 \\
10 & 0.5 & 4 & A12B2 \\
\hline
\end{tabular}

The heterogeneity could be found in both genotype and fluconazole susceptibility for the isolates from patient A8. But most of strains (6/10) belonged to the same genotype (A9B2). Up to 32 fold differences in fluconazole, $\mathrm{MIC}_{50}$ were detected within isolates recovered at the same patient and episode, which is consistent with the results of Lopez-Ribot.

\section{Discussion}

Oral candidiasis is a common side-effect of anticancer chemotherapy for malignant cancer patients (Clarkson et al., 2000; Worthington and Clarkson, 2002). Our results however showed that the frequency of oral Candida carriage was 58\% which was similar with the afore-reported frequency of healthy host. There were 19\% (10/52) patients who had the clinical diagnosis of oral candidiasis. This frequency of oral yeast carriage and oral candidiasis varied according to different
Table 3 Heterogeneity in fluconazole susceptibility for C. albicans isolated from patient A12

\begin{tabular}{lccc}
\hline Strains & $\mathrm{MIC}_{50} / \mu \mathrm{g} \cdot \mathrm{mL}^{-1}$ & $\mathrm{MIC}_{90} / \mu \mathrm{g} \cdot \mathrm{mL}^{-1}$ & Genotype \\
\hline 1 & 0.5 & $>128$ & $\mathrm{~A} 6 \mathrm{~B} 1$ \\
2 & 1 & $>128$ & $\mathrm{~A} 6 \mathrm{~B} 1$ \\
3 & 0.5 & $>128$ & $\mathrm{~A} 6 \mathrm{~B} 1$ \\
4 & 0.5 & $>128$ & $\mathrm{~A} 6 \mathrm{~B} 1$ \\
5 & $>128$ & $>128$ & $\mathrm{~A} 6 \mathrm{~B} 1$ \\
6 & 0.125 & $>128$ & $\mathrm{~A} 6 \mathrm{~B} 1$ \\
7 & $>128$ & $>128$ & $\mathrm{~A} 6 \mathrm{~B} 1$ \\
8 & $>128$ & $>128$ & $\mathrm{~A} 6 \mathrm{~B} 1$ \\
9 & $>128$ & $>128$ & $\mathrm{~A} 6 \mathrm{~B} 1$ \\
10 & 0.125 & $>128$ & A6B1 \\
\hline
\end{tabular}

All the C. albicans isolates from the patient A12 were of genotype A6B1. And they have the same level of $\operatorname{MIC}_{90}\left(>128 \mu \mathrm{g} \cdot \mathrm{mL}^{-1}\right)$, but different $\mathrm{MIC}_{50}$ for fluconazole.

studies. The difference in sampling preparation and position, treatment conditions of the patients, and geographic area may contribute to the diversity of frequency (Rodu et al., 1984; da Costa et al., 2009).

More and more researches indicated that heterogeneity of phenotype and genotype among the population of microorganisms (Wimpenny et al., 2000; Kontoyiannis and Lewis, 2002). It is believed that heterogeneity may play a role in determining the fitness of cell populations, and by producing variant subpopulation of different phenotypes, the cell population can be more suitable for persisting during harsh condition (Bishop et al., 2007;). This is important for the microorganism population which inhabit at a frequently changing environ- 
ment such as oral cavity (McArthur, 2006). Thus the heterogeneity of phenotype and genotype in the oral yeast population should be reasonable. In this study, all the $C$. albicans colonies from the same patients were selected for heterogeneity study. We found that $C$. albicans isolates with different fluconazole MICs and genotypes could coexist in the same oral Candida population, which reinforces this hypothesis.

RAPD is an ideal method for analyzing the $C$. albicans genotype, greatly dependent on the choice of primers and the genomic locus selected. In this research we employed the primer RSD12 and RSD6 for they have been successfully subgrouping the yeast strains. Distinctively different from the other research using primer RSD12, RSD12 in our research only elicited 2 genotypes from among the 205 isolates. The C. albicans strains isolated from different geographic areas may contribute to this phenomenon (Tsang et al., 1995).

From the results of genotypic determinations of Group A patients using RAPD analysis, the multiple colonies from the same patient generally belong to the similar genotype. Although there still had an exception such as strain A8, multiple genotypes with different MIC for fluconazole coexisted in the same population, and most of the strains (6/10) still belonged to the same genotype. Genotypic heterogeneity also could be detected in the population of some patients of Group B, although much fewer isolates from Group B patients. The results indicate that multiple isolates may be reproduced by one or two colonies. The genotypic heterogeneity may be ascribed to the interference of transient strains (Metzgar et al., 1998). But the isolates with the same genotype show different antifungal susceptibility, which reminds us that this hypothesis needs to be further studied.

Resistance of C. albicans isolates to azoles is the most prevalent type of antifungal resistance, also showing great heterogeneity (Lopez-Ribot et al., 1999; Kontoyiannis and Lewis, 2002). Fluconazole resistance in C.albicans can be attributed to overexpression of the drug target (lanosterol demethylase), mutations in the drug target, and most commonly for high-level resistance, overexpression of energy-dependent drug efflux pumps. Our research indicated that although some $C$. albicans population showed great heterogeneity in fluconazole susceptibility, analyzing both $\mathrm{MIC}_{50}$ and $\mathrm{MIC}_{90}$ can be helpful for grasping the whole view of the population. For example, all the isolates from patient $\mathrm{A} 12$ showed very high $\mathrm{MIC}_{90}$ despite a great diversity in $\mathrm{MIC}_{50}$. This fact remind us that comprehensive consideration of $\mathrm{MIC}_{50}$ and $\mathrm{MIC}_{90}$ for azole antifungal drugs susceptibility or some measurement such as agar dilution screening technique (Patterson et al., 1996a; Patterson et al., 1996b) may be useful to provide a more comprehensive assessment and avoid the aberration in clinical practice.

\section{Acknowledgements}

This study was supported by grants from the National Nature Science Foundation of China (No. 30400498).

\section{References}

Akopyanz N, Bukanov NO, Westblom TU, Kresovisch S, Berg DE (1992). DNA diversity among clinical isolates of Helicobacter pylori detected by PCR-based RAPD fingerprinting. Nucleic Acids Res, 20(19): 5137-5142.

Bishop AL, Rab FA, Sumner ER, Avery SV (2007). Phenotypic heterogeneity can enhance rare-cell survival in 'stress-sensitive' yeast populations. Mol Microbiol, 63(2): 507-520.

Brawner DL, Cutler JE (1989). Oral Candida albicans isolates from nonhospitalized normal carriers, immunocompetent hospitalized patients, and immunocompromised patients with or without acquired immunodeficiency syndrome. J Clin Microbiol, 27(6): 13351341.

Cannon RD, Holmes AR, Mason AB, Monk BC (1995). Oral Candida: clearance, colonization, or candidiasis? $J$ Dent Res, 74(5): 1152-1161.

Carderone RA (2002). Candida and Candidiasis. Washington DC: ASM press, pp. 307-326.

Clarkson JE, Worthington HV, Eden OB (2000). Prevention of oral mucositis or oral candidiasis for patients with cancer receiving chemotherapy (excluding head and neck cancer). Cochrane Database Syst Rev, 2: CD 000978.

da Costa KR, Ferreira JC, Komesu MC, Candido RC (2009). Candida albicans and Candida tropicalis in oral candidosis: quantitative analysis, exoenzyme activity, and antifungal drug sensitivity. Mycopathologia, 167(2): 
73-79.

Fell JW (1993). Rapid identification of yeast species using three primers in polymerase chain reaction. Mol Mar Biol Biotechnol, 2( 3): 174-180.

Kleinegger CL, Lockhart SR, Vargas K, Soll DR (1996). Frequency, intensity, species, and strains of oral Candida vary as a function of host age. J Clin Microbiol, 34(9): 2246-2254.

Kontoyiannis DP, Lewis RE (2002). Antifungal drug resistance of pathogenic fungi. Lancet, 359(9312): 11351144.

Lopez-Ribot JL, McAtee RK, Perea S, Kirkpatrick WR, Rinaldi MG, Patterson TF (1999). Multiple resistant phenotypes of Candida albicans coexist during episodes of oropharyngeal candidiasis in human immunodeficiency virus-infected patients. Antimicrob Agents and Chemother, 43(7): 1621-1630.

Mackenzie DW (1962). Serum tube identification of Candida albicans. J Clin Pathol, 15(6): 563-565.

McArthur JV (2006). Microbial ecology: an evolutionary approach. Amsterdam: Academic Press, pp. 270-271.

McCullough MJ, Ross BC, Reade PC (1996). Candida albicans: a review of its history, taxonomy, epidemiology, virulence attributes, and methods of strain differentiation. Int J Oral Maxillofac Surg, 25(2): 136-144.

Mead GM (2002). Management of oral mucositis associated with cancer chemotherapy. Lancet, 359 (9309): 815-816.

Metzgar D, van Belkum A, Field D, Haubrich R, Wills C (1998). Random amplification of polymorphic DNA and microsatellite genotyping of pre- and post-treatment isolates of Candida spp. from human immunodeficiency virus-infected patients on different fluconazole regimens. J Clin Microbiol, 36(8): 2308-2313.

Patterson TF, Kirkpatrick WR, Revankar SG, McAtee RK, Fothergill AW, McCarthy DI, et al. (1996). Comparative evaluation of macrodilution and chromogenic agar screening for determining fluconazole susceptibility of Candida albicans. J Clin Microbiol, 34(12): 3237-3239.

Patterson TF, Revankar SG, Kirkpatrick WR, Dib O, Fothergill AW, Redding SW, et al. (1996). Simple method for detecting fluconazole-resistant yeasts with chromogenic agar. J Clin Microbiol, 34(7): 1794-797.

Qi QG, Hu T, Zhou XD (2005). Frequency, species and molecular characterization of oral Candida in hosts of different age in China. J Oral Pathol Med, 34(6): 352-356.

Rodu B, Griffin IL, Gockerman JP (1984). Oral candidiasis in cancer patients. South Med J, 77(3): 312-314.

Rüchel R, Schaffrinski M, Seshan KR, Cole GT (2000). Vital staining of fungal elements in deep-seated mycotic lesions during experimental murine mycoses using the parenterally applied optical brightener Blankophor. Med Mycol, 38(3): 231-237.

Stewart PS, Franklin MJ (2008). Physiological heterogeneity in biofilms. Nat Rev Microbiol, 6(3): 199-210.

Takasuka T, Baily GG, Birch M, Anderson MJ, Law D, Denning DW (1998). Variation in morphotype, karyotype and DNA type of fluconazole resistant Candida albicans from an AIDS patient. J Infect, 36(1): 57-62.

Tamura M, Watanabe K, Mikami Y, Yazawa K, Nishimura K (2001). Molecular characterization of new clinical isolates of Candida albicans and C. dubliniensis in Japan: analysis reveals a new genotype of $C$. albicans with group I intron. JClin Microbiol, 39(12): 4309-315.

Tsang PC, Samaranayake LP, Philipsen HP, McCulloug M, Reichart PA, Schmidt-Westhausen A, et al. (1995). Biotypes of oral Candida albicans isolates in human immunodeficiency virus-infected patients from diverse geographic locations. J Oral Pathol Med, 24(1): 32-36.

Waltimo TM, Dassanayake RS, Ørstavik D, Haapasalo MP, Samaranayake LP (2001). Phenotypes and randomly amplified polymorphic DNA profiles of Candida albicans isolates from root canal infections in a Finnish population. Oral Microbiol Immunol, 16(2): 106-112.

Wimpenny J, Manz W, Szewzyk U (2000). Heterogeneity in biofilms. FEMS Microbiol Rev, 24(5): 661-671.

Wingard JR (1994). Infections due to resistant Candida species in patients with cancer who are receiving chemotherapy. Clin Infect Dis, 19(Suppl 1): 49-53.

Worthington HV, Clarkson JE (2002). Prevention of oral mucositis and oral candidiasis for patients with cancer treated with chemotherapy: cochrane systematic review. $J$ Dent Educ, 66(8): 903-911.

Xu J, Millar BC, Moore JE, McClurg R, Walker MJ, Evans $\mathrm{J}$, et al. (2002). Comparison of API20C with molecular identification of Candida spp isolated from bloodstream infections. J Clin Pathol, 55(10): 774-777.

\footnotetext{
*Corresponding author: Qing-guo Qi

Address: Oral Medicine, School of Stomatology Shandong University, 44-1 ${ }^{\#}$ Wenhuaxi Road, Jinan 250012, China

Tel: $8653188382213 \quad$ Fax: $8653182950194 \quad$ E-mail: qqg@sdu.edu.cn
} 